\title{
Enhancer cooperativity as a novel mechanism underlying the transcriptional regulation of E-cadherin during mesenchymal to epithelial transition
}

\author{
Hani Alotaibi ${ }^{\text {a,b }}$, M. Felicia Basilicata ${ }^{\mathrm{a}}$, Huma Shehwana ${ }^{\mathrm{c}}$, Tyler Kosowan ${ }^{\mathrm{a}}$, Ilona Schreck ${ }^{\mathrm{a}}$, \\ Christien Braeutigam a ${ }^{\text {, Ozlen Konu }}{ }^{\text {c, Thomas Brabletz }}{ }^{\text {d,e,f,g }}$, Marc P. Stemmler ${ }^{\text {a,d,g,* }}$ \\ a Department of Molecular Embryology, Max-Planck Institute of Immunobiology and Epigenetics, Stuebeweg 51, D-79108 Freiburg, Germany \\ b Izmir Biomedicine and Genome Center, Dokuz Eylül University, Inciralti, 35340 Izmir, Turkey \\ c Department of Molecular Biology and Genetics, Bilkent University, 06800 Ankara, Turkey \\ d Department of Visceral Surgery, University Medical Center Freiburg, Hugstetter Str. 55, D-79106 Freiburg, Germany \\ e Comprehensive Cancer Center Freiburg, University Medical Center Freiburg, D-79106 Freiburg, Germany \\ ${ }^{\mathrm{f}}$ BIOSS Centre for Biological Signaling Studies, Albert-Ludwigs-University Freiburg, D-79104 Freiburg, Germany \\ ${ }^{\mathrm{g}}$ Institute of Experimental Medicine I, Nikolaus-Fiebiger-Center for Molecular Medicine, University Erlangen-Nürnberg, D-91054 Erlangen, Germany
}

\section{A R T I C L E I N F O}

\section{Article history:}

Received 13 October 2014

Received in revised form 6 January 2015

Accepted 24 January 2015

Available online 31 January 2015

\section{Keywords:}

Cadherins

Grhl3

Hnf $4 \alpha$

Transforming growth factor beta

Development

Cancer

\begin{abstract}
A B S T R A C T
Epithelial-mesenchymal transition (EMT) and mesenchymal-epithelial transition (MET) highlight crucial steps during embryogenesis and tumorigenesis. Induction of dramatic changes in gene expression and cell features is reflected by modulation of Cdh1 (E-cadherin) expression. We show that Cdh1 activity during MET is governed by two enhancers at $+7.8 \mathrm{~kb}$ and at $+11.5 \mathrm{~kb}$ within intron 2 that are activated by binding of Grhl3 and $\mathrm{Hnf} 4 \alpha$, respectively. Recruitment of Grhl3 and $\mathrm{Hnf} 4 \alpha$ to the enhancers is crucial for activating Cdh1 and accomplishing MET in non-tumorigenic mouse mammary gland cells (NMuMG). Moreover, the two enhancers cooperate via Grhl3 and Hnf $4 \alpha$ binding, induction of DNA-looping and clustering at the promoter to orchestrate E-cadherin re-expression. Our results provide novel insights into the cellular mechanisms whereby cells respond to MET signals and re-establish an epithelial phenotype by enhancer cooperativity. A general importance of our findings including MET-mediated colonization of metastasizing tumor cells is suggested.
\end{abstract}

(C) 2015 Elsevier B.V. All rights reserved.

\section{Introduction}

Many key steps during embryogenesis result in the formation of new cell types with unique features. They become morphologically visible when individual cells or tissues are generated by cell delamination during a process called epithelial-mesenchymal transition (EMT) or by cell clustering and re-epithelialization during mesenchymal-epithelial transition (MET). EMT is required for mesoderm formation, neural crest cell delamination, fibrosis and wound healing, but is also aberrantly activated during tumorigenesis when cancer cells start to disseminate, invade and form metastases [1-3]. Common to all types of EMT are cytoskeletal rearrangements resulting in loss of cell polarity and adherent morphology combined with increased migration. Gene expression signatures are changing dramatically with a major impact on the repertoire of cell adhesion molecules, especially of $\mathrm{Ca}^{2+}$-dependent adhesion molecules, the cadherins [4]. Strikingly, changes in cellular characteristics during a bona fide EMT are to a large extent dependent

\footnotetext{
* Corresponding author. Tel.: +499131 85 29101; fax: + 4991318529341.

E-mail address: marc.stemmler@fau.de (M.P. Stemmler).
}

on the downregulation of E-cadherin (E-cad) and the activation of $\mathrm{N}$-cadherin ( $\mathrm{N}$-cad), regulated by the EMT program [1].

MET is considered as the reverse process of EMT and also originates from embryogenesis $[5,6]$. Here, mesenchymal cells acquire epithelial characteristics including loss of $\mathrm{N}$-cad and activation of E-cad expression [1-3]. In addition to orchestrating morphogenetic events during embryogenesis the process of MET is utilized by disseminating tumor cells and is required for colonization and formation of metastases at distant sites $[1,2,7]$. In vitro MET is necessary for somatic cell reprogramming. During the generation of induced pluripotent stem (iPS) cells, MET precedes the activation of the endogenous loci of transcription factors of the core pluripotency network [8]. This is in part established by the activation of E-cad expression via direct binding of exogenous Klf4 to specific sites at the promoter [9]. Interestingly, E-cad supports the initiation of MET and increases reprogramming efficiency [10]. In contrast, E-cad depletion results in loss of pluripotency and decreases the potential for reprogramming [11]. All these findings depict EMT, MET and a tightly regulated expression of cadherins as crucial during many different processes in development and disease. 
Hallmarks of transitions between epithelial and mesenchymal states include changes in the cadherin repertoire. An essential step of EMT is the downregulation of E-cad whereas its expression is rapidly restored during MET. Many different extracellular signals are known to trigger EMT leading to activation of intracellular EMT-inducers like Snail, Slug, Twist, Zeb1, Zeb2 and others [1,2]. In agreement with a required fast downregulation of E-cad, all of these transcription factors are in fact repressors of E-cad expression and they all bind to several evolutionary conserved E-boxes present in the proximal promoter [12-18]. However, the immediate activation of the Cdh1 locus during MET can only in part be explained by the downregulation and release of these repressors. We found that the Cdh1 promoter alone, including all known E-box elements, is insufficient to confer strict cell type specificity. In contrast, intron 2 carries sufficient information for proper E-cad expression $[19,20]$. In particular, we identified sequences in the proximal $15 \mathrm{~kb}$ of the $45 \mathrm{~kb}$ spanning intron 2, that showed cell type specific gene activation, mainly in the endoderm [20]. Moreover, an endogenous $C d h 1$ allele lacking the entire intron 2 failed to activate expression of a reporter, showing that intron 2 is essential for the initiation and maintenance of E-cad expression during development [19]. Although intron 2 has emerged as an essential regulator of $C d h 1$ expression, very little is known about the molecular determinants controlling the transcriptional activity of the locus. Besides Klf4, it has been shown that Grainyhead-like 2 (Grhl2), a mammalian homolog of Drosophila grainyhead, controls epithelial differentiation in several epithelia and at uretic bud formation by regulating E-cad via binding to an element in intron 2 [21].

Here, we sought to identify novel enhancers within intron 2 of Cdh1 and to discover the corresponding transcription factors that mediate gene activation. We used non-tumorigenic mouse mammary gland epithelial cells (NMuMG) that undergo a reversible EMT by transforming growth factor $\beta$ (TGF $\beta$ ) treatment. We identified two potent enhancers that cooperate in establishing prompt and robust E-cad expression during the induction of MET. Furthermore, we provide evidence that transcriptional activity from these enhancers is mediated by two novel E-cad activators, Grhl3 and $\mathrm{Hnf} 4 \alpha$, which emerge as regulators of MET in NMuMG cells.

\section{Materials and methods}

\subsection{Cell culture and induction of EMT and MET}

NMuMG; non-tumorigenic mouse mammary gland cell line, Hepa1-6; mouse hepatoma cell line, CMT; mouse polyploid carcinoma cell line and HEK-293; human embryonic kidney cell line were obtained from ATCC. Cells were maintained in DMEM (Gibco) and 10\% fetal bovine serum (FBS) at $37^{\circ} \mathrm{C}, 10 \% \mathrm{CO}_{2}$. NMuMG medium was supplemented with $10 \mu \mathrm{g} / \mathrm{ml}$ insulin (Sigma). EMT induction of NMuMG cells was done by applying $5 \mathrm{ng} / \mathrm{ml} \mathrm{TGF \beta 3}$ (PeproTech) for $72 \mathrm{~h}$. The reversible transition to the epithelial state (MET) was induced by TGFß3 withdrawal, washing the plates twice with PBS and incubation for additional $72 \mathrm{~h}$ in fresh medium.

\subsection{Luciferase reporter assays}

Reporter plasmids and expression vectors used here are available in the Supplemental Experimental Procedures. Cells in 48-well plates were transfected with plasmid DNAs using X-tremeGENE 9 transfection reagent (Roche). Transfection was carried out with $100 \mathrm{ng}$ DNA containing $5 \mathrm{ng}$ pRL-TK (Promega) to normalize for transfection efficiency. We normalized to equal molarity of the plasmid to use equal copies of reporter plasmid. Absolute DNA content was equalized by addition of promoter-less plasmid DNA. In experiments with TGF 33 , the drug was added at the time point of transfection. Cells recovering from TGF 33 treatment (PT) were transfected upon TGFB3 withdrawal and measured after 3 days of recovery. $20 \mathrm{ng}$ of
Grhl3, Grhl2, Grhl1 and Hnf4a expression plasmid or empty vector (mock) were transfected. Luciferase reporter activity was measured using Dual-Glo Luciferase Assay (Promega) in a Centro LB 960 luminometer (Berthold Technologies). Firefly luciferase reporter values were normalized to those of the Renilla luciferase control. Fold induction was calculated relative to the values of the empty vector or vehicle control samples.

\subsection{Generation of transgenic embryos}

Animal husbandry and all experiments were performed according to the German Animal Welfare guidelines and approved by the local authorities. The reporter construct TG3 (Wt) or the one with mutated Grhl3 binding site 7.8b (Mut) were injected into oocytes as described [20].

\subsection{Chromosome conformation capture (3C)}

The protocol for 3C was described previously [22]. In brief, cells were grown in the presence or absence of $5 \mathrm{ng} / \mathrm{ml}$ TGFß3 and allowed to recover after TGF 33 withdrawal and cross-linked with $1 \%$ formaldehyde for $10 \mathrm{~min}$ at room temperature. After lysis on ice for $10 \mathrm{~min}$ in lysis buffer (10 mM Tris- $\mathrm{HCl}, \mathrm{pH} 7.5 ; 10 \mathrm{mM} \mathrm{NaCl}$; $5 \mathrm{mM} \mathrm{MgCl} 2 ; 0.1 \mathrm{mM}$ EGTA; $1 \times$ complete protease inhibitor), nuclei were incubated with TaqI for 90 min at $37^{\circ} \mathrm{C}$. Purified DNA was used together with BAC RP23-262 N14 DNA containing the murine Cdh1 locus for qPCR analysis. Primers used are presented in Supplementary Table S4.

\subsection{Chromatin immunoprecipitation (ChIP)}

ChIP was performed as reported previously [23] with minor modifications as depicted in the Supplemental Experimental Procedures using Grhl3 (S-19, Santa Cruz), Hnf4 $\alpha$ (H-171, Santa Cruz), p300 (C-20, Santa Cruz), H3K9Ac (ab4441, Abcam), H3K4me1 (ab8895, Abcam) and a rabbit control IgG (sc-2027, Santa Cruz) antibodies.

\subsection{Co-immunoprecipitation and immunoblotting}

Immunoprecipitation was done as described in detail in the Supplemental Experimental Procedures using $1 \mathrm{mg}$ nuclear extracts and $25 \mu \mathrm{l}$ of anti-HA affinity matrix (Roche) or $4 \mu \mathrm{g}$ of anti-FLAG antibody (M2, Sigma) coupled to Protein-G Dynabeads.

\subsection{Statistical analysis}

Statistical significance was determined by performing the Student's $t$-test using the normalized values of each test sample compared to the normalized value of the control, using a 95\% confidence interval; p-values less than 0.05 were considered significant. Data are presented as the mean of at least three independent experiments, done in triplicates. ChIP and 3C were carried out three times, qPCR was performed in triplicates. Error bars represent standard error of the mean.

\section{Results}

\subsection{Intron 2 of Cdh1 harbors conserved and potent enhancers}

We reported previously that intron 2 of $C d h 1$ is essential for proper E-cad expression $[19,20]$. In order to analyze this large DNA sequence (>40 kb) for putative enhancers we focused on evolutionary conserved sequences. We rationalized that a conserved expression pattern is reflected by a conserved regulatory mechanism utilizing the same enhancers conserved in highly similar genomic regions among different species [24]. To identify such putative enhancers, we used the mVista 


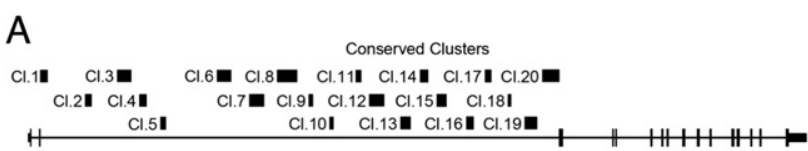

B

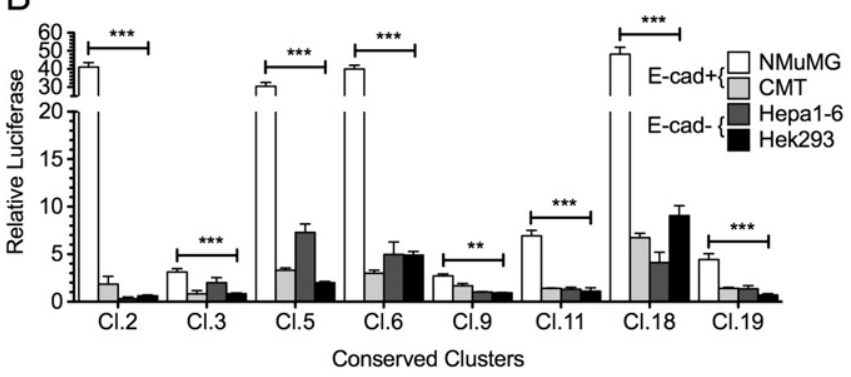

C

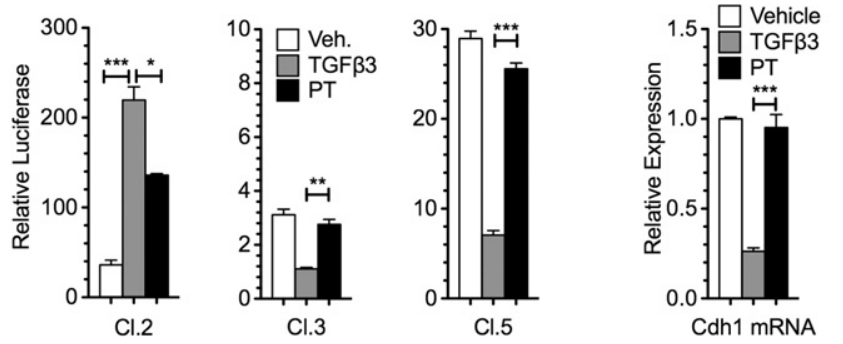

Fig. 1. Identification of functional conserved sequences within Cdh1 intron 2. (A) A map of the conserved clusters is shown in relation to the $C d h 1$ gene, location and size of clusters are in scale. $\mathrm{Cl} .1-\mathrm{Cl} .20$ indicate individual clusters and refer to the Luciferase constructs below (Supplementary Table S1). (B) Luciferase assay of constructs harboring conserved clusters in E-cad expressing and E-cad negative cell lines. Relative luciferase is calculated as fold induction relative to the empty vector pGL4.23. (C) Reporter gene activity of selected clusters in NMuMG cells in response to three days of vehicle and TGFB3 treatment as well as after three days of TGFB3 withdrawal (post-treatment, PT). (D) qRT-PCR analysis of endogenous E-cad mRNA in response to TGFB3. *, $\mathrm{p}<0.05$; ${ }^{* *}, \mathrm{p}<0.01$; ***, $\mathrm{p}<0.001$.

multiple alignment tool [25] for cross-species sequence comparison of the Cdh1 locus, and compared the genome of mouse to those of human, rhesus, dog, and horse (Supplementary Fig. S1A, Supplementary Table S1). We identified several sequences with more than $70 \%$ conservation in a $150 \mathrm{bp}$ window consistent with our previous analysis [19]. These conserved clusters were distributed over the entire intron 2 (Fig. 1A). Individual clusters were cloned into a luciferase reporter vector, upstream of a minimal promoter (Supplementary Fig. S1B) and their enhancing potential was analyzed by measuring luciferase activity. We used two E-cad expressing (non-tumorigenic mammary gland and rectal polyploid carcinoma cells, NMuMG and CMT, respectively) and two E-cad negative cell lines (immortalized embryonic kidney and hepatoma cells, HEK-293 and Hepa1-6). To identify the most important sequences, the clusters were sorted out with respect to importance for E-cad regulation by two criteria. First we selected conserved sequences with stronger activity in cells expressing E-cad compared to those which are E-cad negative (Fig. 1B). The analysis showed that 8 out of the 20 clusters had stronger activity in the E-cad + cell line NMuMG when compared to the E-cad- cell lines (Fig. 1B, Supplementary Fig. S1B). None of the clusters showed strong activity in CMT cells, indicating a more complex mode of regulation exceeding the limitation of this technique (Fig. 1B, Supplementary Fig. S1B). As a second criterion 8 clusters with a higher activity in E-cad + cell lines were tested for changes in reporter activity during EMT in response to TGFB3 treatment and during MET after TGF 33 withdrawal (post-treatment, PT) in NMuMG cells (Fig. 1C, Supplementary Fig. S1C). In total three conserved sequences were found to be responsive to TGF 33 , but the reporter activity of only two ( $\mathrm{Cl} .3$ and $\mathrm{Cl} .5$ ) recapitulated the expression of $\mathrm{E}$-cad in response to TGF 33 , whereas $\mathrm{Cl} .2$ showed inverse correlation (Fig. 1C and D).

\subsection{Grhl3 but neither Grhl1 nor Grhl2 activates Cdh1 via an intronic enhancer in Cl.3 in NMuMG cells}

We used the MatInspector tool from the Genomatix Suite [26] to screen $\mathrm{Cl} .3$ and $\mathrm{Cl} .5$ for transcription factor binding sites. We identified a recognition motif in $\mathrm{Cl} .3$ for grainyhead-like transcription factors, proteins known to be required for pattern specification and tissue development in several species including Drosophila [27-30]. In mice it has been recently shown that Grhl2 is controlling E-cad expression during MET of uretic bud formation by binding to two highly conserved Grhl motifs in tandem within intron 2 located at $7.8 \mathrm{~kb}$ (7.8a and 7.8b) relative to the transcription start site (TSS) [21] (Supplementary Fig. S2A). We searched for presence of Grhl binding sites in intron 2 and restricted the analysis to the proximal $11 \mathrm{~kb}$, which we previously identified as being sufficient to faithfully recapitulate endodermal E-cad expression in transgenic reporter mice [20]. In total we identified 17 putative Grhl binding sites, five of which are located within the $11 \mathrm{~kb}$ fragment (Fig. 2A, Supplementary Table S2). We tested whether the recognition motifs were also functional Grhl2 binding sites and utilized for Cdh1 regulation in NMuMG cells by luciferase reporter assays. The architecture of the locus was maintained in the reporter constructs by cloning the luciferase reporter between the promoter (nucleotides from -1490 to +1 ) and the entire region between $+0.1 \mathrm{~kb}$ and $+11 \mathrm{~kb}$ of the $\mathrm{Cdh} 1$ gene (Cl.1-4) (Fig. 2A). To our surprise, we found that Cl.1-4 was not activated by neither Grhl2 nor Grhl1. In contrast, ectopic Grhl3 expression increased reporter gene activity 4-fold (Fig. 2B, Supplementary Fig. S2F).

We also created several deletion constructs for analysis of individual binding sites that contained one or two of the four conserved clusters from the analysis in Fig. 1. In addition to the original $11 \mathrm{~kb}$ fragment, containing $\mathrm{Cl} .1-4$ and sites $1.4,2.4,7.8 \mathrm{a}, 7.8 \mathrm{~b}$ and 9.6, only deletion constructs containing $\mathrm{Cl} .3$ were significantly activated by Grhl3 (Fig. 2B). Including downstream sequences up to $16 \mathrm{~kb}$ containing $\mathrm{Cl} .1-5$ and sites 10.7, 12.7 and 13.7 did not significantly increase reporter gene activity (Supplementary Fig. S2B and C). Furthermore, we introduced point mutations in the Grhl3 binding sites at 7.8a, 7.8b and 9.6 (Supplementary Fig. S2D), and found that the mutation at site 7.8b already reduced the basal activity, indicating that endogenous Grhl3 is no longer able to bind and activate the construct. Moreover, this mutation completely abolished the Grhl3-dependent upregulation (Fig. 2C) and resulted in impaired regulation of the reporter during TGF 33 treatment (Fig. 2D). In contrast, mutating site 7.8a had only moderate effects and the construct with a mutation at site 9.7 retained full activity in response to Grhl3 (Fig. 2C, Supplementary Fig. S2C). This suggested a possible role for Grhl3 in regulating transcription from the enhancer at site $7.8 \mathrm{~b}$ in response to TGF 33 .

In order to assess the functionality of this enhancer and the requirement for Grhl3-dependent regulation in vivo independent of MET events, we used the mouse transgenic lacZ-reporter TG3, corresponding to Cl.1-4 [20]. We introduced a point mutation in the Grhl3 binding site at 7.8b and analyzed the reporter expression in the developing mouse embryo at E11.5. In agreement with our previous findings, embryos injected with the wildtype reporter ( $\mathrm{Wt}, \mathrm{n}=9 / 9 \mathrm{X}$-gal positive embryos) were characterized by $\beta$-gal expression in the endoderm of the pharynx, esophagus, lung, stomach and pancreas, reflecting an expression pattern similar to that of E-cad. To our surprise, this expression was absent in embryos carrying the mutant construct (Mut, $\mathrm{n}=0 / 16 \mathrm{X}$-gal positive embryos), indicating an important role for the Grhl3 motif in regulating the expression of E-cad in endodermal epithelia of the developing mouse embryo (Fig. 2E, and Supplementary Fig. S2E). 
A

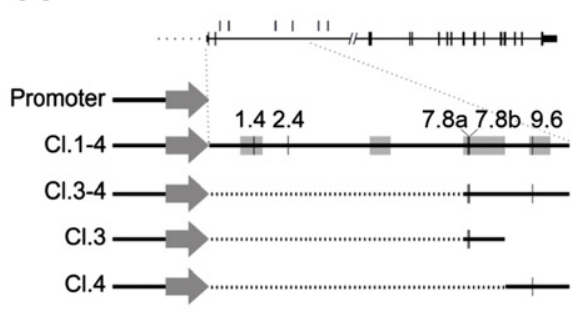

C

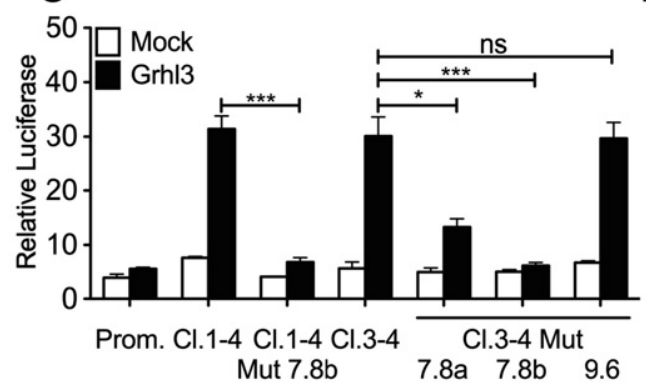

B

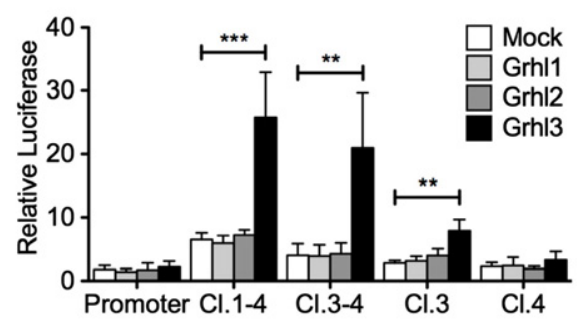

D

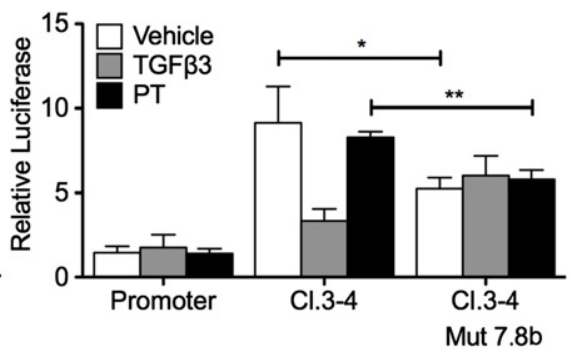

E

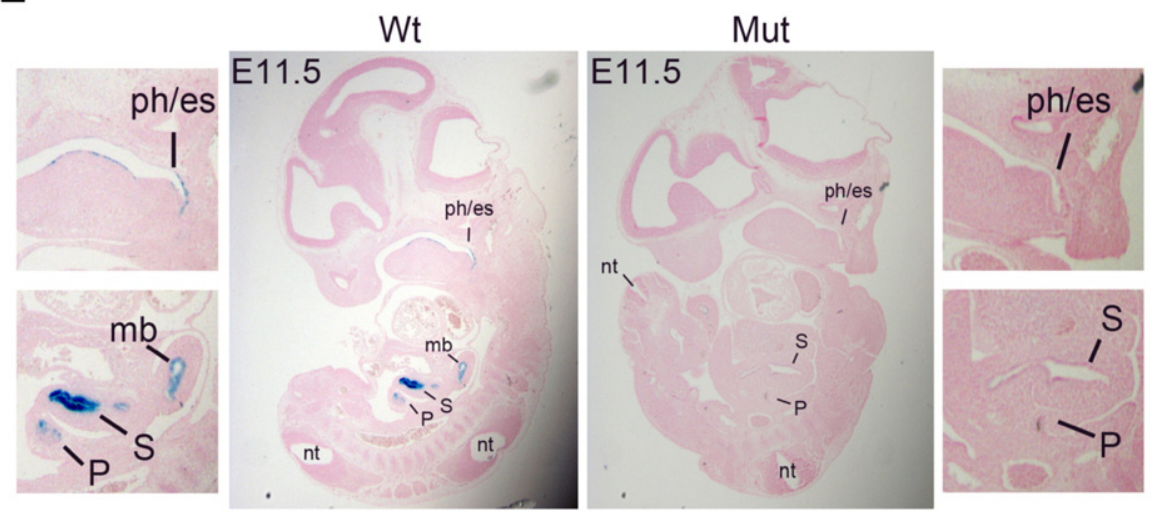

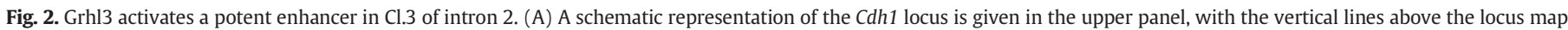

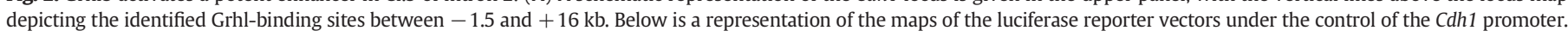

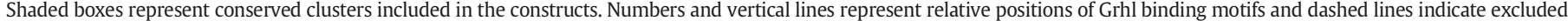

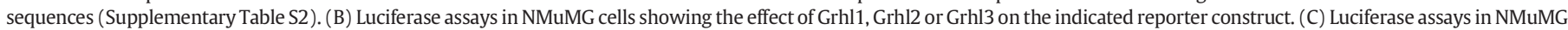

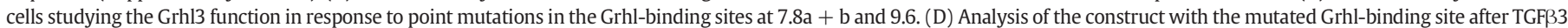

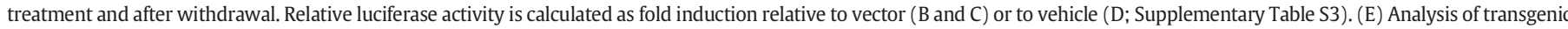

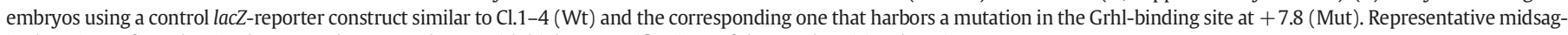
ittal sections of X-gal stained E11.5 embryos are shown with higher magnifications of the X-gal positive domains.

\subsection{Grhl3 binding to E-cad chromatin occurs during mesenchymal to epithelial transition}

When we investigated the presence of Grhl3 on the Cdh1 chromatin in untreated NMuMG cells by ChIP as an indication of direct binding, we could not detect significant enrichment of Grhl3 on any of the predicted sites, including the functional site at $7.8 \mathrm{~b}$ under steady state conditions (Fig. 3A, vehicle). We then took advantage of the ability of these cells to undergo TGFß-dependent reversible EMT. TGFß3 treatment for $72 \mathrm{~h}$ induced EMT, indicated by downregulation of E-cad and upregulation of Vimentin. Upon withdrawal of TGF 33 the cells undergo MET resulting in re-expression of E-cad (Supplementary Fig. S3A and B). We investigated a possible enrichment of Grhl3 on E-cad during MET after TGF33 withdrawal. We found that Grhl3 was significantly enriched at two of the Grhl3 sites (sites 2.4 and $7.8 \mathrm{a}+\mathrm{b}$ ) and also at the TSS $72 \mathrm{~h}$ post-treatment (Fig. 3A, PT), but not in cells treated with vehicle control or TGF33 (Fig. 3A).
In an effort to mechanistically address the observed Grhl3-dependent regulation of E-cad during MET, we investigated the enhancer-promoter interaction. We designed $3 \mathrm{C}$ experiments and compared the relative crosslinking frequencies in NMuMG cells in response to EMT-MET stimuli. Changes in chromosome conformation (DNA looping) can be depicted by measuring crosslinking frequencies of intermolecular ligation products, which represent enhancer-promoter interactions [22]. We used several primers located within intron 2 (Fig. 3B, Supplementary Table S4) and performed the qPCR analysis using a common anchoring primer near the promoter (RT1). We detected elevated crosslinking frequencies with primers corresponding to the location of the enhancers at 2.4 and 7.8a $+\mathrm{b}$ (FI2 and FG2; fragments 3 and 6 respectively) compared to primers located elsewhere between +1 and $+16 \mathrm{~kb}$ (Fig. 3B). Importantly, the crosslinking frequencies of cells undergoing MET were significantly higher than of those treated with TGFB3 or vehicle, suggesting that such enhancers were in close proximity to the promoter. These results are supporting the ChIP experiments, suggesting that in the initial 


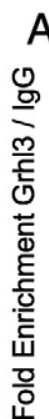

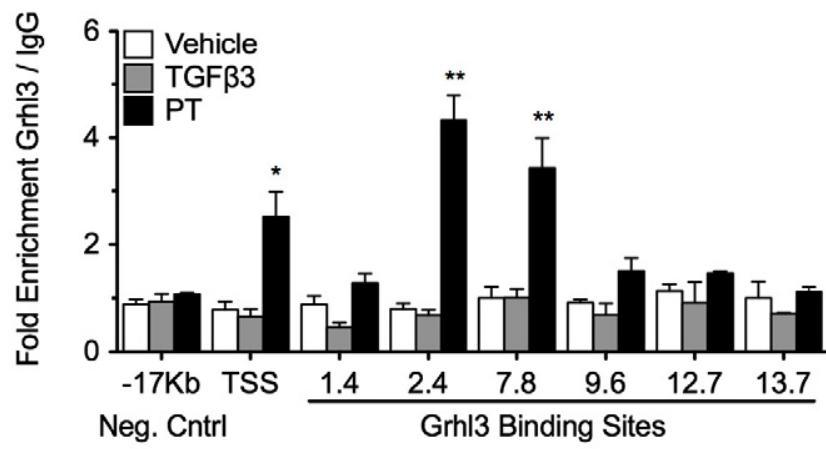

B
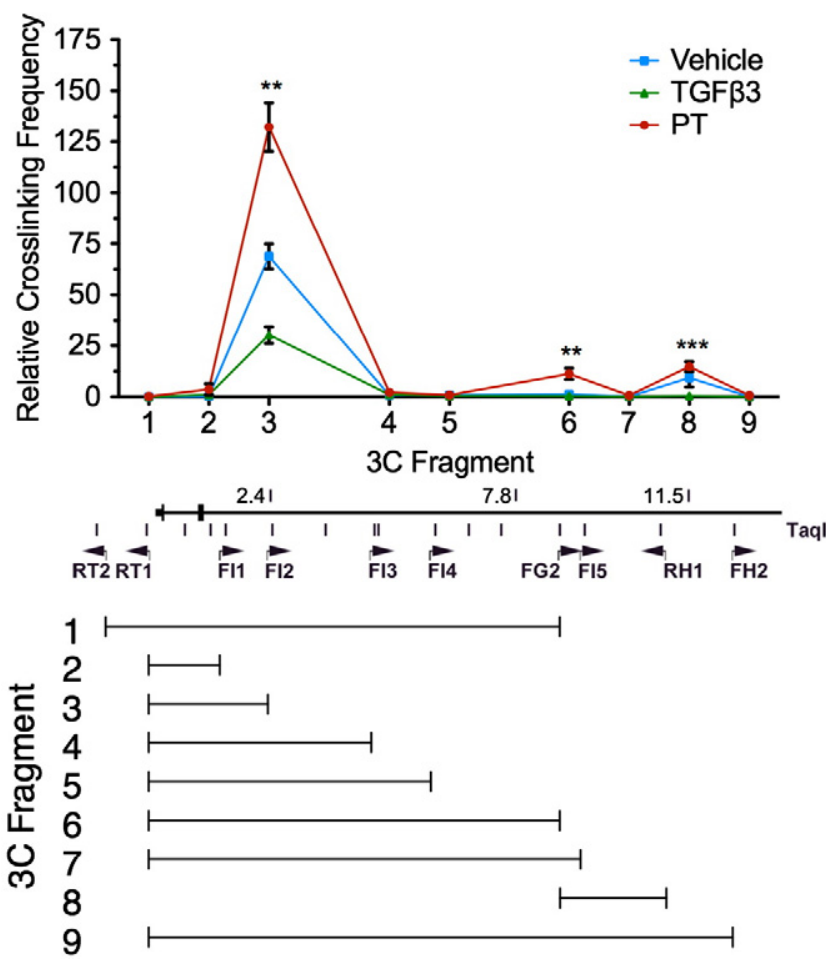

Fig. 3. Grhl3 is enriched at the intronic enhancers during mesenchymal-epithelial transition (MET). (A) ChIP with anti-Grhl3 antibodies in NMuMG cells undergoing EMT-MET in response to TGF33 treatment. Analysis of Grhl-binding sites in comparison to a non-related control sequence $(-17 \mathrm{~kb})$ shows specific enrichment at the $\mathrm{Cl} .3$ enhancer containing binding site +7.8 and at a novel site at +2.4 as well as at the transcriptional start site (TSS; Supplementary Table S5). (B) Chromosome conformation capture (3C) experiment of the Cdh1 locus in response to TGFB3. The schematic representation of the Cdh1 locus indicating the location of the enhancers (vertical lines above) the location of TaqI restriction sites (vertical lines below), and the location of the primers used (arrow heads represent orientation). The capped lines below indicate the primer pair used to amplify each $3 C$ fragment (Supplementary Table S4 and S5).

phase after TGFB3 withdrawal, Grhl3 is recruited to the enhancer. This may lead to an interaction of the Grhl3-DNA enhancer complex with the basal transcription machinery assembled at the promoter. Alternatively, binding of a preassembled Grhl3/basal transcription machinery/ co-factor complex is stabilized by Grhl3-DNA binding at the enhancer. Both scenarios are in line with a gradual increase in Cdh1 mRNA levels after TGFB3 withdrawal (Fig. 1D). No evidence for Grhl3 binding to the putative site at 2.4 was found by luciferase reporter assays in NMuMG cells (Supplementary Fig. S2C). A mutation at site 7.8b alone was sufficient to abrogate Grhl3-dependent luciferase reporter activation and also the lacZ-reporter activity in transgenic embryos. In contrast, $3 \mathrm{C}$ and
ChIP data revealed that this enhancer is utilized and recruits Grhl3 to the site at 2.4 in a context-dependent manner. Specifically, it is active during MET after TGFB3 withdrawal. Some of the primers used in this experiment were in close proximity to the conserved sequence $\mathrm{Cl} .5$ from Fig. 1 (primers FH2 and RH1; fragment 9). Although there was no detectable interaction between this conserved sequence and the promoter in this context, we were able to detect a significant interaction with the enhancer at 7.8a $+\mathrm{b}$ (primers RH1 and FG2; fragment 8), which was also a TGF33 responsive interaction detectable during MET (Fig. 3B, PT), suggesting a possible interplay between these two enhancers at 11.5 and $7.8 \mathrm{a}+\mathrm{b}$

We then analyzed the effect of Grhl3 downregulation on E-cad expression in NMuMG cells using several Grhl3 siRNAs. We found that two of the three analyzed siRNAs resulted in more than $80 \%$ downregulation of Grhl3 (Fig. 4A). This reduction in Grhl3 expression resulted in a significant decrease in E-cad expression levels only in cells recovering from TGFB3 treatment (Fig. 4A), but not in cells treated with the vehicle (Supplementary Fig. S3C). We also noticed that with increasing

\section{A}

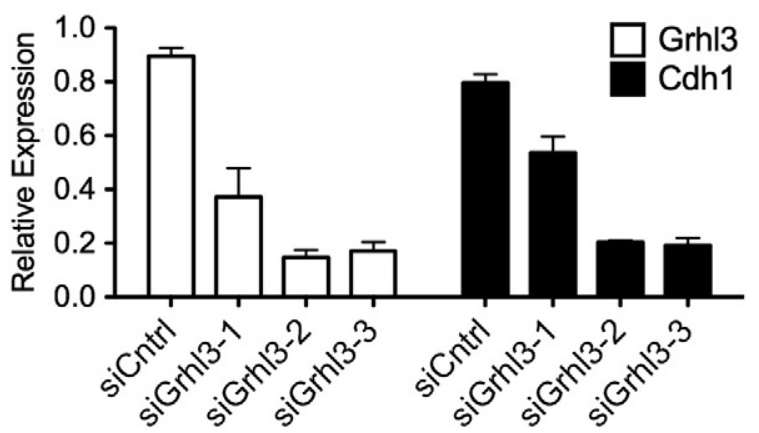

B

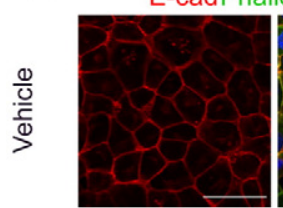

E-cadPhalloidin DAPI Vimentin Phalloidin DAPI

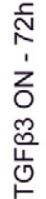
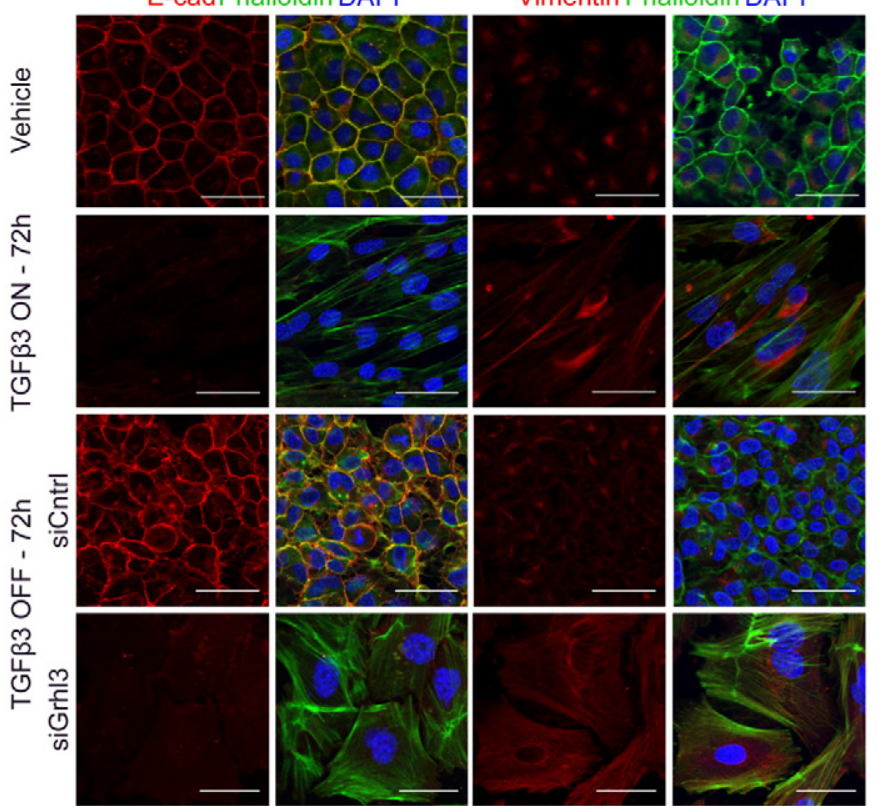

Fig. 4. Grhl3 depletion results in E-cad downregulation and a failure of MET. (A) qPCR analysis of mRNA of NMuMG cells recovering from TGFB3 treatment after Grhl3 knockdown using three different siRNAs and the effect on E-cad mRNA levels (Supplementary Table S6). (B) Immunofluorescence labeling and phalloidin staining of NMuMG cells visualizing the change in expression and intracellular distribution of E-cad, Vimentin and Actin during TGFB3 treatment and withdrawal. Actin distribution (Phal, green) and detection of E-cad (red) and Vimentin (red) is shown. Nuclei are labeled with DAPI. Scale bar, $50 \mu \mathrm{m}$. 
efficiencies of Grhl3 silencing, the effect on E-cad downregulation was more apparent (Fig. 4A, compare siGrhl3-1 and siGrhl3-2). To our surprise, the siRNA mediated loss of Grhl3 expression after TGFB3 withdrawal blocked MET (Fig. 4B). This was evident by the absence of E-cad expression, elevated Vimentin expression and the arrangement of cortical actin in stress fibers, reminiscent of continuous TGFB3-mediated EMT (Fig. 4B, Supplementary Fig. S3D and E). A similar block of MET can also be observed in cells transfected with siRNAs targeting E-cad during the recovery from TGFB3 treatment (Supplementary Fig. S3E). This indicated that Grhl3 function in this context is indispensable for proper MET. In part this is accomplished by Grhl3 positively regulating the expression of E-cad, which is essential for proper transition to the epithelial state after TGF33 withdrawal.

\subsection{Hnf $4 \alpha$ specific regulation of E-cad via $\mathrm{Cl} .5$ in a TGFß-dependent manner}

Our analysis showed that the mode of action of the enhancer within $\mathrm{Cl} .3$ is mainly mediated by Grhl3 during MET of NMuMG cells and during embryogenesis in the endoderm. Next, we investigated the contribution of $\mathrm{Cl} .5$ to $C d h 1$ gene regulation in mammary epithelial cells before and after TGFB3 treatment. We searched the sequences of $\mathrm{Cl} .5$ for the presence of putative transcription factor binding sites using the MatInspector tool of the Genomatix suite [26]. Among the several hits obtained we were particularly interested in a highly conserved recognition motif for the orphan nuclear receptor hepatocyte nuclear factor 4 alpha $(\mathrm{Hnf} 4 \alpha)$ located at 11.5 relative to the TSS (Fig. 5A, Supplementary Fig. S1A). Hnf $4 \alpha$, which is faithfully coexpressed with E-cad, was previously shown to regulate several adhesion molecules (including E-cad) during fetal liver organogenesis [31]. Using luciferase reporter assays, we first tested whether Hnf $4 \alpha$ could activate Cl.5 in NMuMG cells. We found that indeed $\mathrm{Hnf} 4 \alpha$ was able to exert a robust enhancing effect on the E-cad $\mathrm{Cl} .5$ reporter construct. Two different mutations in the putative $\mathrm{Hnf} 4 \alpha$ binding site decreased the basal activity of this construct and inhibited its $\mathrm{Hnf} 4 \alpha$-dependent activation (Fig. 5B). Furthermore, we found that the two mutations in the $\mathrm{Hnf} 4 \alpha$ consensus motif were sufficient to abolish recovery of the reporter activity upon withdrawal of TGFB3 (Fig. 5C), suggesting that this recovery is Hnf4 $\alpha$ dependent.
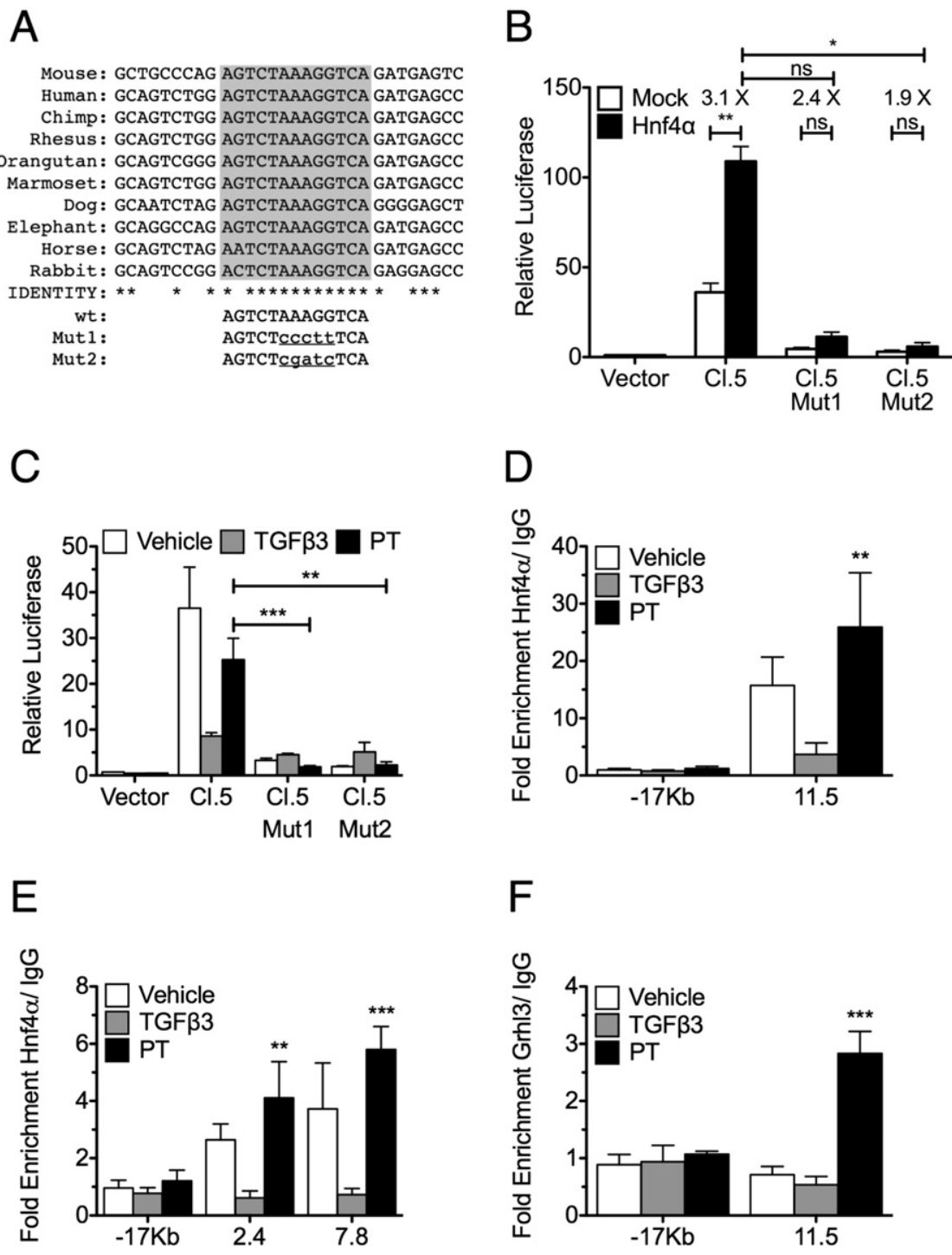

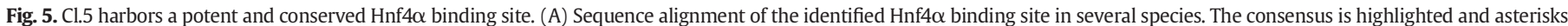

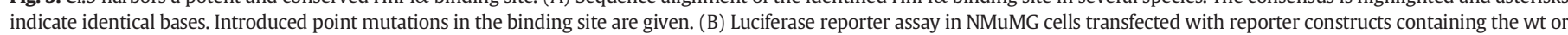

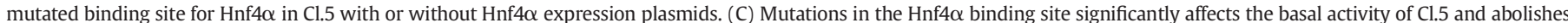

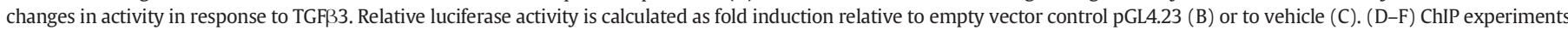

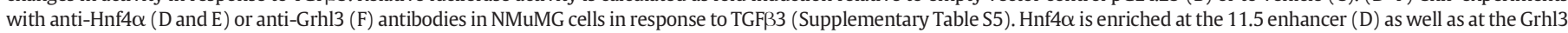
bound enhancers at 2.4 and 7.8 (E) and Grhl3 is bound to the 11.5 enhancer (F). 
The remaining $\operatorname{Hnf} 4 \alpha$-dependent increase in activity of the mutant reporter constructs was not significant.

Using ChIP experiments we detected significant enrichment of Hnf $4 \alpha$ on this novel enhancer in NMuMG cells in a TGF $\beta$-dependent fashion (Fig. 5D). In contrast to Grhl3 enrichment on the $\mathrm{Cl} .3$ intronic sites, $\mathrm{Hnf} 4 \alpha$ recruitment to the chromatin was detectable already in untreated NMuMG cells under steady state conditions, suggesting a distinct function for this enhancer. Hnf $4 \alpha$ disappeared from $\mathrm{Cl} .5$ during TGFB3-mediated EMT and Cdh1 downregulation and it was enriched 1.5 fold $72 \mathrm{~h}$ after TGF33 withdrawal (Fig. 5D). To our surprise, we also detected significant enrichment of $\mathrm{Hnf} 4 \alpha$ at both Grhl3 enhancers at sites 2.4 and 7.8a + b (Fig. 5E, Supplementary Fig. S4C). A similar enrichment of Grhl3 was identified at the $\mathrm{Hnf} 4 \alpha$ binding site at 11.5 (Fig. 5F). This indicated a possible interaction between these two enhancers in agreement with the $3 \mathrm{C}$ results that showed looping of $\mathrm{Cl} .5$ to the region of $\mathrm{Cl} .3$.

\subsection{Grhl3 and Hnf4 $\alpha$ functionally interact at the intronic enhancers}

The Hnf $4 \alpha$ enrichment at the Grhl3 enhancers and the interaction observed with 3C experiments intrigued us to look for a potential genetic or physical interaction between the two factors and between $\mathrm{Cl} .3$ and Cl.5. First we performed luciferase reporter assays using $\mathrm{Cl} .3$ and studied the effect of overexpressing Hnf $4 \alpha$ on Grhl3 function. We observed a significant increase in reporter activity when $\mathrm{Hnf} 4 \alpha$ was introduced together with Grhl3 compared to the Grhl3-specific activation alone (Fig. 6A). While $\operatorname{Hnf} 4 \alpha$ alone did not increase the reporter activity, the observed effect of Hnf4 $\alpha$ over Grhl3 was specific, since no increase was visible when we introduced Cebp $\alpha$ as control (data not shown).

Next, we used siRNAs to downregulate Hnf $4 \alpha$ expression (Supplementary Fig. S4A), and found that the absence of $\mathrm{Hnf} 4 \alpha$ resulted in a modest but significant decrease in the Grhl3-dependent activation of the reporter, compared to the control (Fig. 6B). We wanted to determine if a similar effect could be observed on Cdh1 chromatin. For this purpose, we first treated NMuMG cells with TGFB3 for $72 \mathrm{~h}$ and at the time of TGFB3 withdrawal, cells were transfected with siRNAs targeting Hnf4a. After $72 \mathrm{~h}$ post-treatment we performed ChIP using Grhl3 specific antibodies and measured the Grhl3 enrichment at the previously identified Grhl3 sites and at the promoter. As anticipated, loss of $\mathrm{Hnf} 4 \alpha$ expression dramatically reduced the Grhl3 enrichment at the Cdh1 intronic enhancers as well as at the promoter (TSS), although the latter does not contain consensus sites for Hnf $4 \alpha$ and Grhl3 (Fig. 6C, Supplementary Fig. S4E). This was accompanied by a modest but significant decrease in the levels of E-cad mRNA as measured by qPCR (Fig. 6D). We hypothesized that the Hnf $4 \alpha$-dependent recruitment of Grhl3 to intronic enhancers may be due to a physical interaction between the two proteins and validated this by co-immunoprecipitation experiments. We transfected HA-Hnf $4 \alpha$ into NMuMG cells during MET after TGF33 removal. Anti-Grhl3 immunoprecipitation revealed a complex of $\mathrm{Hnf} 4 \alpha$ and Grhl3 as indicated by the co-immunoprecipitation of HA-Hnf $4 \alpha$ (Supplementary Fig. S4B). Physical interaction was also confirmed in HEK-293 cells ectopically expressing tagged versions of the two proteins. To abolish DNA-mediated indirect coupling of the two transcription factors we digested DNA by benzonase treatment of cell lysates. Immunoprecipitation of either $\mathrm{HA}-\mathrm{Hnf} 4 \alpha$ or flag-Grhl3 resulted in specific co-precipitation of the corresponding binding partner (Fig. 6E).

p300 is a crucial co-factor of $\mathrm{Hnf} 4 \alpha$-mediated gene regulation and found in the PolII-transcription initiation complex at many promoters [32,33]. p300 was detected on both enhancers in control cells and at the TSS with highest enrichment at the Grhl3 enhancer at 7.8a + b. Importantly, these levels were diminished upon depletion of $\mathrm{Hnf} 4 \alpha$ (Fig. 6F), indicating that p300 recruitment was $\mathrm{Hnf} 4 \alpha$-dependent. Moreover, we addressed the effect of $\mathrm{Hnf} 4 \alpha$ depletion on the architecture of $C d h 1$ chromatin by looking at an active histone mark (acetylation at Lys9 of H3, H3K9Ac, Fig. 6G) and one that is associated with enhancers (monomethylation at Lys4 of H3, H3K4me1, Supplementary
Fig. S4C and D). We found that both marks were enriched at both enhancers and at the TSS in comparison to a control region. Upon Hnf4a knockdown both marks were significantly reduced in those regions (Fig. 6G, Supplementary Fig. S4D). Since both Grhl3 and Hnf4 $\alpha$ were found also at the promoter (Figs. 3A and 6C, Supplementary Fig. S4C), we investigated whether unidentified non-canonical binding sites are present at the promoter. Neither Grhl3 nor Hnf4 $\alpha$ were increasing Cdh1 promoter-only reporter gene activity, indicating that both factors are only indirectly linked to the TSS (Supplementary Fig. S4E). Taken together, our results suggest that $\mathrm{Hnf} 4 \alpha$ is crucial for stabilizing the chromatin structure in a permissive state, enabling proper binding of Grhl3 to the intronic enhancers for a robust upregulation of E-cad expression.

\subsection{Grhl3 contributes to the regulation of Hnf4 $\alpha$ expression during MET}

To analyze how Grhl3 and $\mathrm{Hnf} 4 \alpha$ are connected to orchestrate the re-expression of E-cad during MET, we studied E-cad, Grhl3 and Hnf4 $\alpha$ mRNA levels in a time course experiment after the withdrawal of TGFB3 (Fig. 7A). We noticed that the expression of Grhl3 was restored rapidly and was first to reach $50 \%$ of its initial level within $24 \mathrm{~h}$. While Hnf4 $\alpha$ expression was also increasing, it fell behind the levels of Grhl3 and reached 50\% of the initial level 6-12 h later. E-cad expression levels increased steadily but were reaching original levels later than Grhl3 and Hnf4 $\alpha$ after $72 \mathrm{~h}$ (Fig. 7A). Based on these data, we hypothesized that besides its crucial role in the re-expression of E-cad, Grhl3 may also be responsible for the upregulation of $\mathrm{Hnf} 4 \alpha$ which in turn assists Grhl3 at Cdh1 enhancers for appropriate re-establishment of E-cad levels.

The pattern of $\mathrm{Hnf} 4 \alpha$ expression suggested a level of regulation by Grhl3. We analyzed the expression of $\mathrm{Hnf} 4 \alpha$ following the downregulation of Grhl3. Our results confirmed that in response to the depletion of Grhl3, a significant decrease in Hnf $4 \alpha$ mRNA levels was observed (Fig. 7B). Hnf $4 a$ is activated from two alternative promoters (P1 and P2 in Fig. 7C) resulting in at least six different splice variants [34] with the major isoforms Hnf $4 \alpha 1$ containing Exon $1 \mathrm{~A}$ and $\mathrm{Hnf} 4 \alpha \mathrm{7}$ containing Exon 1D derived from the P1 and P2 promoters, respectively $[35,36]$. We found a Grhl3 recognition motif in P1 at -339 bp and a very wellconserved one in P2 at $-29 \mathrm{~kb}$ (Fig. 7C). Anti-Grhl3 ChIP revealed that Grhl3 was significantly enriched at P2 but not at P1 in cells recovering from TGF 33 , similar to the enrichment at the Cdh1 enhancers (Fig. 7D). This indicated that isoforms containing Exon 1D are regulated by Grhl3, which is in agreement with previous findings showing that P2 is the active promoter in NMuMG cells controlling mainly $\mathrm{Hnf} 4 \alpha 8$ expression [37]. In summary our results suggest that in response to MET signals Grhl3 and Hnf4 $\alpha$ are immediately upregulated, recruited together with p300 to the Cdh1 locus and cooperatively initiate E-cad expression. In parallel Grhl3 is directly activating Hnf4a which accelerates the MET program.

\subsection{Grhl3 and Cdh1 expression is correlated in mouse mammary cells and} in human breast cancer cell lines

Finally, we aimed to evaluate whether our findings are also reflected in expression data sets in mouse mammary tissue and have implications during tumor progression in human breast cancer samples. We first investigated whether Grhl1-3, Hnf4a and Cdh1 gene expression values were correlated across normal mouse mammary tissue subpopulations (i.e., stroma, basal, luminal, epithelial and total) using data from GSE40877. We found that Grhl1, Grhl2, Grhl3 and Cdh1 were all highly and positively correlated with each other (Fig. S5A; Supplementary Table S8). Next, we analyzed data sets of established human breast cancer cell lines from the CCLE (Cancer Cell Line Encyclopedia) A significant positive correlation among GRHL1-3 and CDH1 was observed (Fig. S5B; Supplementary Table S8). Microarray data sets of a fibroblast/breast cancer cell co-culture system (GSE41678) revealed that the CDH1 expressing Cal51 breast cancer cell line highly expressed GRHL3 and/or HNF4A in monoculture and in co-culture with fibroblasts (Fig. S5C). In 
A

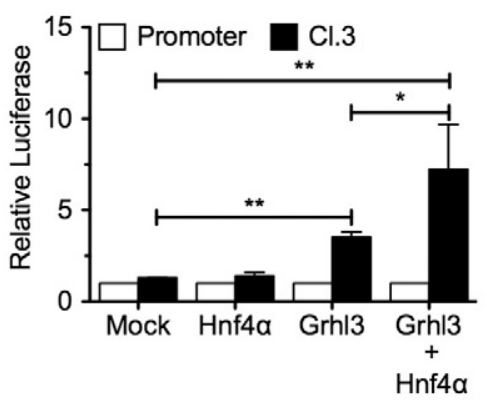

C

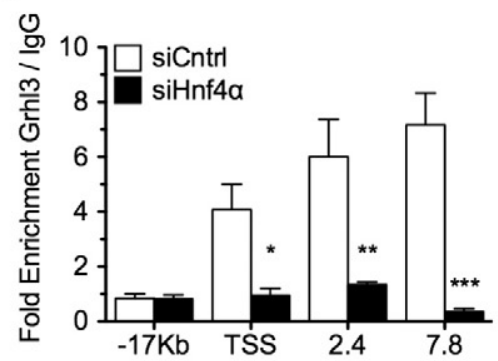

B

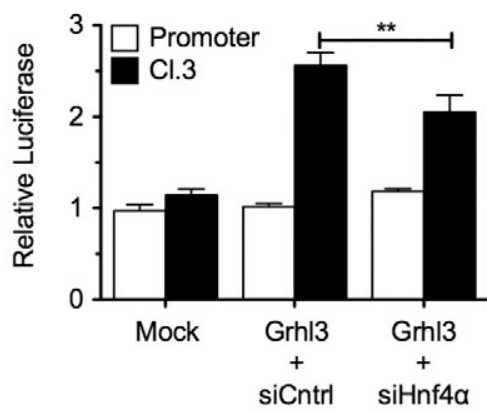

$\mathrm{D}$

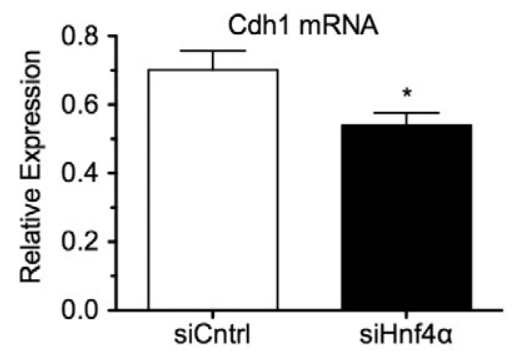

$E$

IP: HA

HA-Hnf4a flag-Grhl3 + HA-matrix + +
IP: flag

--+ HA-Hnf4a $++\quad-$

-+ + flag-Grhl3 + + - +

- - $\operatorname{lgG}+-\quad-$

anti-flag - + -
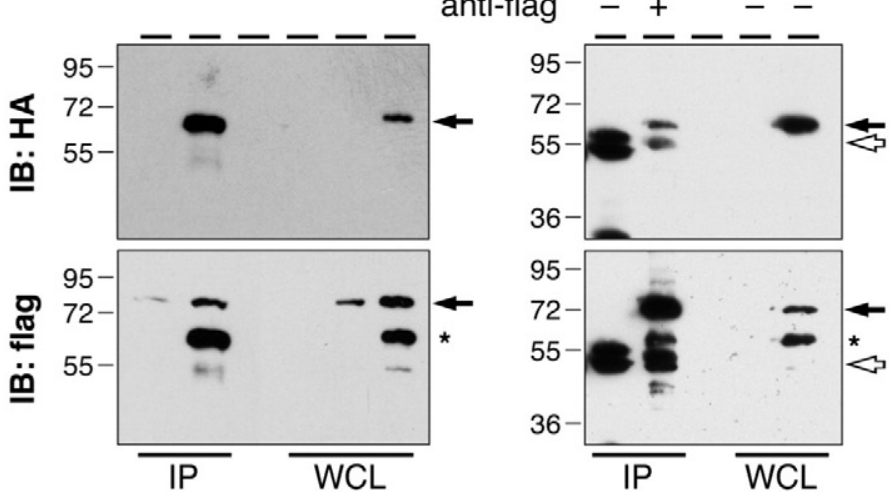

$\mathrm{F}$

G
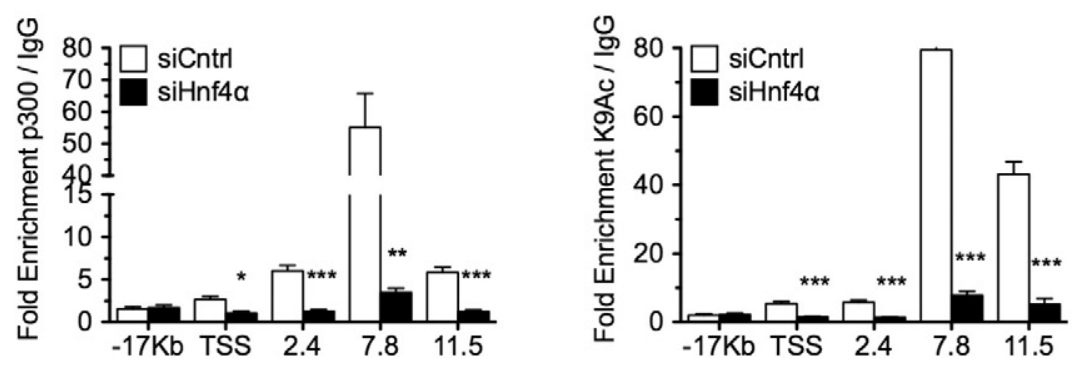

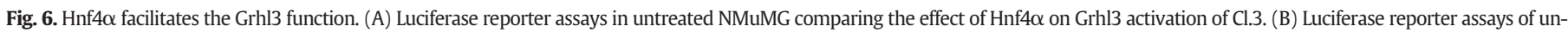

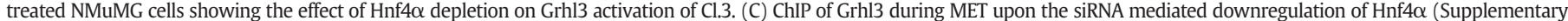

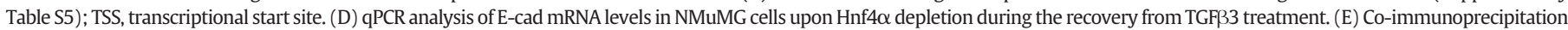

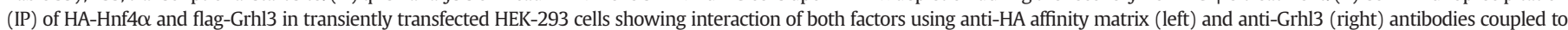

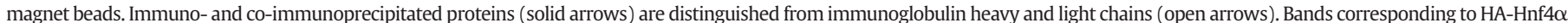

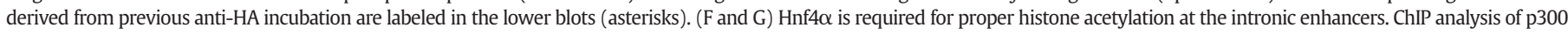
$(\mathrm{F})$ and $\mathrm{H} 3 \mathrm{~K} 9 \mathrm{Ac}(\mathrm{G})$ enrichment in response to Hnf4 $\alpha$ depletion during the recovery from TGF33 treatment (Supplementary Table S5). 
A

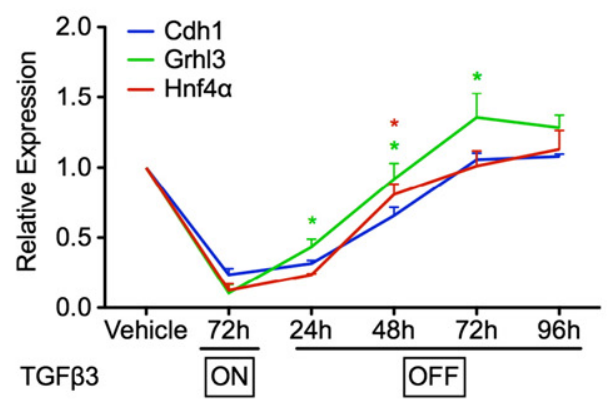

C

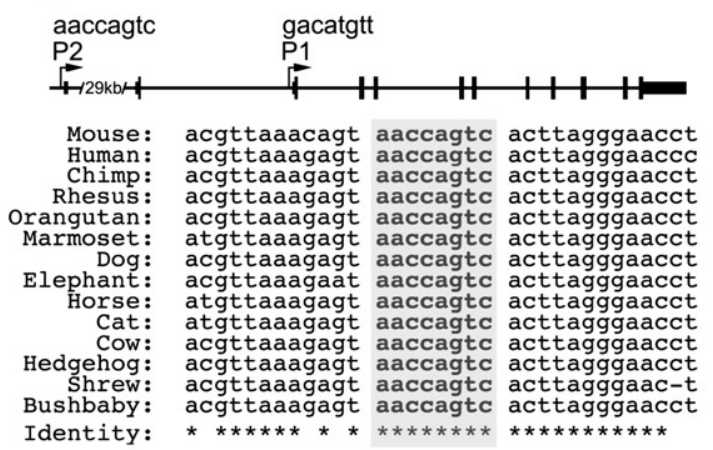

B

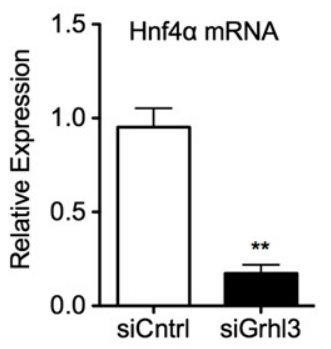

D

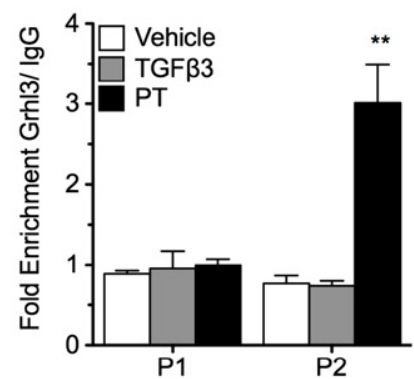

$E$

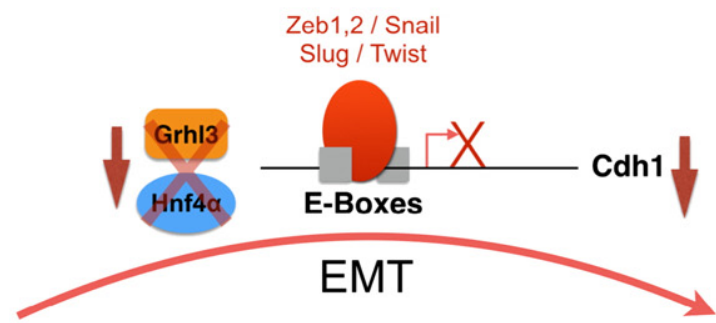

Epithelial

Mesenchymal
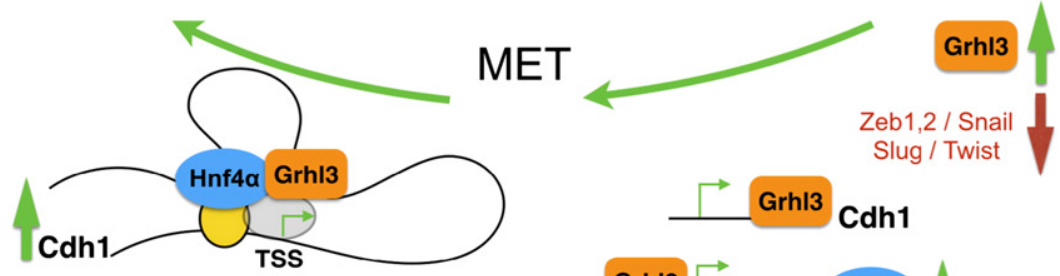

Grhl3

\section{Cdh1}

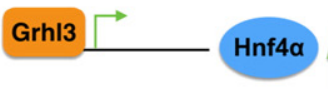

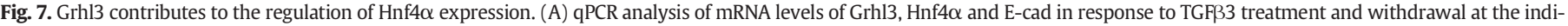

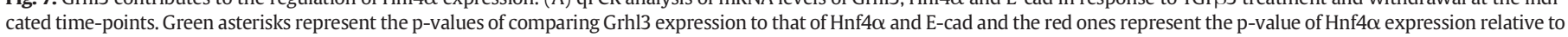

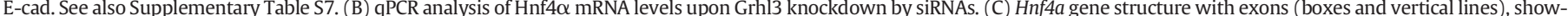

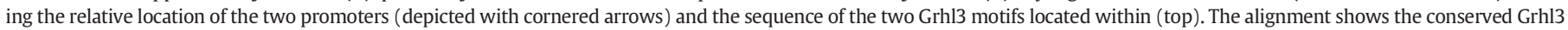

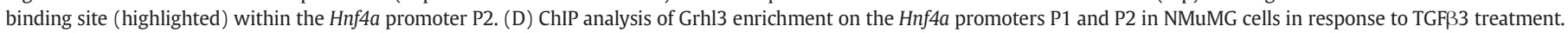

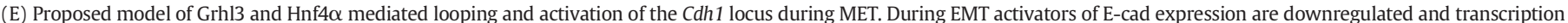

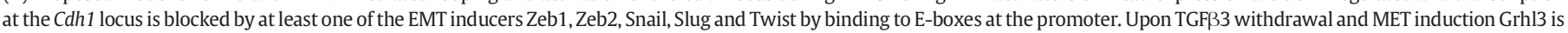

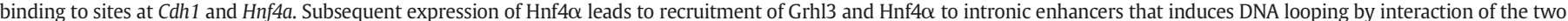

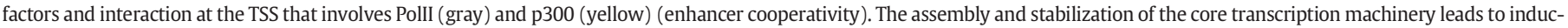
tion of E-cad expression. Up- and downregulation is indicated by vertical green and red arrows, respectively.

contrast, basal like MDA-MB-231 breast cancer cells expressed all three genes at low levels regardless of the culturing methodology (Supplementary Fig. S5C). GRHL3 expression was correlated with GRHL1, GRHL2, CDH1 as well as with HNF4A (Fig. S5C; Supplementary Table S8). In line with our previous findings, these data support that $C d h 1$ expression is mainly dependent on Grhl3, whereas $\operatorname{Hnf} 4 \alpha$ might contribute to the control of the Cdh1 locus in a tissue and context-dependent manner, especially during EMT/MET processes. In contrast to MET in NMuMG cells, based on the correlated expression in the analyzed data sets also Grhl1 and Grhl2 might be involved in Cdh1 locus control in steady-state conditions as well. 


\section{Discussion}

In early embryonic development proper cell adhesion mediated by E-cad is crucial for the shaping of the embryo proper and for establishing the embryonic-maternal interface of the placenta [38-41]. During these processes EMT and MET programs are initiated and marked by the switching of cadherin gene expression, suggesting that a tight and dynamic control of the Cdh1 locus is mandatory. Proper promoter function is dependent on cis-regulatory elements located in the $47 \mathrm{~kb}$ intron 2.

By comparing sequences of the mammalian species mouse, man, rhesus monkey, dog and horse followed by functional analyses, we identified novel $C d h 1$ enhancers at $+7.8 \mathrm{~kb}$ and $+11.5 \mathrm{~kb}$ downstream of the TSS. These enhancers are important for Cdh1 gene activation during MET in NMuMG cells. Our data indicate that Grhl3 and Hnf4 $\alpha$ are mediating this activation by binding to the enhancers at $+7.8 \mathrm{~kb}$ and $+11.5 \mathrm{~kb}$, respectively. Abolishing the binding site for Grhl3 at $+7.8 \mathrm{~kb}$ resulted in loss of expression of a lacZ-reporter construct in transgenic mouse embryos suggesting that this site plays a crucial role for E-cad expression in vivo as well. Moreover, Grhl3 and Hnf4 $\alpha$ were bound to each other and cooperated during gene activation. In addition to their own binding sites they were also found to occupy each other's enhancers. Despite of the lack of binding sites within the E-cad promoter, they were also detected at the TSS, suggesting looping of DNA to complex all involved transcription factors with the basal transcription machinery (Fig. 7E).

The family of grainyhead transcription factors has been first described in Drosophila. Later three homologs of a family of six members were identified in vertebrates: grainyhead-like 1 (Grhl1), Grhl2 and Grhl3 [42-44]. In Drosophila, grainyhead (grh) is driving postembryonic neuroblast function and proliferation in part by affecting DE-cadherin expression $[28,44]$. In mammals, the expression is largely confined to epithelia. Although their expression only partially overlaps, overall at least one member is coexpressed with E-cad $[19,42,43]$. Knockout mice were described for all three, with Grhl2 showing the most severe phenotype as embryos die at E11.5 [27,29,30,45]. Each knockout show common defects in neural tube closure at specific sites, establishment of epithelial function, differentiation and barrier formation, all of which depend on proper E-cad function. Similarly, $\mathrm{Hnf} 4 \alpha$, an orphan member of the nuclear receptor superfamily, is also coexpressed with E-cad in several tissues such as liver, intestine and kidney [19,46]. Zygotic depletion of $\mathrm{Hnf} 4 \alpha$ results in a pregastrulation defect due to its role in the visceral endoderm [47]. Conditional ablation of Hnf $4 \alpha$ in the embryonic liver primordium or in the colon displays defects in epithelial specification and in organ function and homeostasis $[31,48]$. Cdh1 was one prominent gene that was significantly reduced upon $\mathrm{Hnf} 4 \alpha$ depletion in the liver, suggesting a general mechanism of $C d h 1$ transcriptional control by $\mathrm{Hnf} 4 \alpha$ in epithelia. Their role in epithelial specification, an overlapping expression with E-cad and the phenotypes of knockout mice, renders $\mathrm{Grhl}$ factors and $\mathrm{Hnf} 4 \alpha$ prime candidates for the regulation of Cdh1.

Are Grhl factors redundant for E-cad regulation? It was recently shown by Werth and colleagues that Grhl2 acts similarly to grh in Drosophila development in regulating Cdh1 transcription $[21,28]$. Loss of Grhl2 reduces E-cad expression in several epithelia including the gut tube and the otic vesicle. In mouse inner medullary collecting duct cells (mIMCD-3) and embryonic lungs Grhl2 binds to the same enhancer at $7.8 \mathrm{a}+\mathrm{b}$ that was occupied by Grhl3 in our experiments $[21,28]$. Interestingly, Grhl2 did not show an activating effect in our system (Fig. 2B, Supplementary Fig. S3D). Only Grhl3 was able to upregulate the luciferase reporter and Grhl3 depletion during MET abolished the re-expression of E-cad and the re-establishment of an epithelial state (Fig. 4, Supplementary Fig. S3D). Although Grhl3 is highly expressed in the skin and mammary bud epithelium [19,42,43], a comprehensive expression analysis in mammary gland epithelium is lacking. Microarray re-analysis revealed that Grhl1-3 are correlated with $C d h 1$, and thus are enriched in epithelial/luminal cell populations in mouse mammary tissues. We here used NMuMG cells mainly as a simple model for reversible EMT and MET to study Cdh1 control using TGF $\beta$ as one of many triggers to induce EMT. Hence, although we obtained strong support of a cooperativity mechanism of the two transcription factors in Cdh1 regulation, generalization is limited. However, also in epithelia during embryonic development and independent of MET the Grhl3-binding site is crucial for proper $C d h 1$ gene activation. Since this site would potentially be bound by other factors, we cannot completely rule out that in mouse embryos our transgenic reporter construct is regulated also by other Grhl family members, conferring reduced $C d h 1$ reporter gene activity independent of Grhl3. However, expression analysis showed that Grhl3 and Grhl1 rather than Grhl2 are expressed at high levels in the stomach epithelium [29,42]. Additionally, we did not observe Cdh1 transactivation by Grhl2 in NMuMG and other cell lines, indicating tissue-specificity of individual Grhl factors. It will be interesting to analyze whether Grhl2 in mIMCD-3 cells is interacting in a similar fashion with $\mathrm{Hnf} 4 \alpha$ or different regulators of $C d h 1$ as does Grhl3.

A general regulation of $C d h 1$ by Grhl3 and $\mathrm{Hnf} 4 \alpha$ independent of MET is very likely. First, loss of Hnf $4 \alpha$ in the liver or the intestinal epithelium of the colon substantially reduced E-cad expression due to direct interaction of $\operatorname{Hnf} 4 \alpha$ with a site upstream of the TSS $[31,48]$. Second, the siRNA mediated removal of Grhl3 resulted in 30\% decrease in E-cad mRNA levels also in a variety of epithelial cell lines including untreated NMuMG, CMT, CSG, P19, ESCs and keratinocytes (Supplementary Fig. S3C). Lastly, reporter gene activity in the endoderm of transgenic embryos was depending on the Grhl3 recognition motif. This indicates that Grhl3 is regulating E-cad levels not only during MET events.

Previous data suggested that the complexity of $C d h 1$ gene regulation is governed by interplay of multiple cis-regulatory elements dispersed throughout the locus. We now provide evidence to how this mode of transcriptional control is achieved in molecular terms by the cooperation of two distal enhancers separated by $4 \mathrm{~kb}$. Recently, a novel type of super-enhancers has been identified in embryonic stem cells. They represent clusters of normal enhancers defined by continuous several $\mathrm{kb}$ long stretches occupied by core transcription factors of pluripotency [49]. The presence of super-enhancers at Cdh1 intron 2 cannot be excluded, but the detection of distinct DNaseI hypersensitive sites at each conserved cluster supports the presence of individual small enhancers (not shown). Long-range interaction of distant cis-regulatory elements and DNA looping is a general mechanism to control expression [50]. Binding to the pre-assembled transcriptional machinery is then activated by factors bound to the distal enhancers for efficient transcription [51]. Enhancer cooperativity was shown for other loci, e.g. the $\beta$-globin locus [52] and the MMP13 locus by LEF1 [53]. Using 3C analysis Vakoc et al. showed that GATA-1 and FOG-1 are required to establish the physical interaction of the locus control region and the $\beta$-major globin promoter [52]. Enhancer cooperativity during MET may reflect the need for immediate E-cad upregulation to efficiently enter an epithelial state. This enhancer cooperativity which utilizes Grhl3 and $\mathrm{Hnf} 4 \alpha$ may also affect other epithelial genes. The detected correlation between the expression of CDH1, GRHL3 and HNF4A in many cancer cell lines, breast cancer specimens as well as lung cancer (Supplementary Fig. S5 and data not shown), suggests a general mode of regulation. It is tempting to speculate that we identified a general mechanism that is also acting on Cdh1 in circulating tumor cells once they activate MET and start colonization during metastasis formation.

In summary, we identified how Grhl3 is orchestrating E-cad expression during MET. External stimuli like TGF 3 induce EMT leading to Hnf $4 \alpha$ and Grhl3 downregulation and activation of transcription factors of the ZEB (Zeb1, Zeb2), Snail (Snail, Slug) and bHLH (Twist) families which results in shutdown of E-cad transcription. Upon reversion of the process e.g. by withdrawal of TGF 3 EMT transcription factors are downregulated and Grhl3 expression is initiated. To facilitate MET Grhl3 acts on both Hnf4a and Cdh1 transcriptional activation by binding 
to the corresponding binding sites. To fully activate E-cad expression, $\mathrm{Hnf} 4 \alpha$ is recruited to the binding site at 11.5 of the $C d h 1$ locus. Here, it forms a complex with Grhl3, p300 and probably with other proteins to connect proximal and distal cis-regulatory elements by induction of a DNA loop. This facilitates the assembly of a functional transcription machinery complex at the promoter. $\mathrm{Hnf} 4 \alpha$ and Grhl3 cooperatively bind to their cognate binding sites supporting each other's recruitment. Once the MET process is completed, Grhl3 is released from the Hnf4a promoter and also partially from the $C d h 1$ promoter at site $7.8 \mathrm{~b}$ and steady-state E-cad expression requires only $\mathrm{Hnf} 4 \alpha$ (Fig. 7E).

Supplementary data to this article can be found online at http://dx. doi.org/10.1016/j.bbagrm.2015.01.005.

\section{Funding}

This work was supported by the Max-Planck Society and the Deutsche Forschungsgemeinschaft SFB850 TP A4.

\section{Transparency document}

The Transparency document associated with this article can be found, in the online version.

\section{Acknowledgements}

The Hnf $4 \alpha$ expression construct was kindly provided by Mary Weiss. We thank Robert Kuhnert, Kati Hansen and Jessica Pfannstiel for excellent technical assistance. We are grateful to Drs. Rolf Kemler Andreas Hecht, Jochen Maurer, Mehmet Ozturk and Uygar Tazebay for critically reading the manuscript and helpful discussions.

\section{References}

[1] J.P. Thiery, H. Acloque, R.Y. Huang, M.A. Nieto, Epithelial-mesenchymal transitions in development and disease, Cell 139 (2009) 871-890.

[2] T. Brabletz, To differentiate or not-routes towards metastasis, Nat. Rev. Cancer 12 (2012) 425-436.

[3] R. Kalluri, R.A. Weinberg, The basics of epithelial-mesenchymal transition, J. Clin. Invest. 119 (2009) 1420-1428.

[4] M.P. Stemmler, Cadherins in development and cancer, Mol. BioSyst. 4 (2008) 835-850.

[5] B. Li, Y.W. Zheng, Y. Sano, H. Taniguchi, Evidence for mesenchymal-epithelial transition associated with mouse hepatic stem cell differentiation, PLoS ONE 6 (2011) e17092.

[6] D. Vestweber, R. Kemler, P. Ekblom, Cell-adhesion molecule uvomorulin during kidney development, Dev. Biol. 112 (1985) 213-221.

[7] T. Brabletz, A. Jung, S. Reu, M. Porzner, F. Hlubek, L.A. Kunz-Schughart, R. Knuechel, T. Kirchner, Variable beta-catenin expression in colorectal cancers indicates tumor progression driven by the tumor environment, Proc. Natl. Acad. Sci. U. S. A. 98 (2001) 10356-10361.

[8] R. Li, J. Liang, S. Ni, T. Zhou, X. Qing, H. Li, W. He, J. Chen, F. Li, Q. Zhuang, B. Qin, J. Xu, W. Li, J. Yang, Y. Gan, D. Qin, S. Feng, H. Song, D. Yang, B. Zhang, L. Zeng, L. Lai, M.A. Esteban, D. Pei, A mesenchymal-to-epithelial transition initiates and is required for the nuclear reprogramming of mouse fibroblasts, Cell Stem Cell 7 (2010) 51-63.

[9] T. Chen, D. Yuan, B. Wei, J. Jiang, J. Kang, K. Ling, Y. Gu, J. Li, L. Xiao, G. Pei, E-cadherinmediated cell-cell contact is critical for induced pluripotent stem cell generation, Stem Cells 28 (2010) 1315-1325.

[10] T. Redmer, S. Diecke, T. Grigoryan, A. Quiroga-Negreira, W. Birchmeier, D. Besser, E-cadherin is crucial for embryonic stem cell pluripotency and can replace OCT4 during somatic cell reprogramming, EMBO Rep. 12 (2011) 720-726.

[11] I. Bedzhov, H. Alotaibi, M.F. Basilicata, K. Ahlborn, E. Liszewska, T. Brabletz, M.P. Stemmler, Adhesion, but not a specific cadherin code, is indispensable for ES cell and induced pluripotency, Stem Cell Res. 11 (2013) 1250-1263.

[12] E. Batlle, E. Sancho, C. Franci, D. Dominguez, M. Monfar, J. Baulida, A. Garcia De Herreros, The transcription factor snail is a repressor of E-cadherin gene expression in epithelial tumour cells, Nat. Cell Biol. 2 (2000) 84-89.

[13] J. Yang, S.A. Mani, J.L. Donaher, S. Ramaswamy, R.A. Itzykson, C. Come, P. Savagner, I. Gitelman, A. Richardson, R.A. Weinberg, Twist, a master regulator of morphogenesis, plays an essential role in tumor metastasis, Cell 117 (2004) 927-939.

[14] A. Cano, M.A. Perez-Moreno, I. Rodrigo, A. Locascio, M.J. Blanco, M.G. del Barrio, F. Portillo, M.A. Nieto, The transcription factor snail controls epithelial-mesenchymal transitions by repressing E-cadherin expression, Nat. Cell Biol. 2 (2000) 76-83.

[15] V. Bolos, H. Peinado, M.A. Perez-Moreno, M.F. Fraga, M. Esteller, A. Cano, The transcription factor Slug represses E-cadherin expression and induces epithelial to mesenchymal transitions: a comparison with Snail and E47 repressors, J. Cell Sci. 116 (2003) 499-511.
[16] J. Comijn, G. Berx, P. Vermassen, K. Verschueren, L. van Grunsven, E. Bruyneel, M. Mareel, D. Huylebroeck, F. van Roy, The two-handed E-box binding zinc finger protein Sip1 downregulates E-cadherin and induces invasion, Mol. Cell 7 (2001) 1267-1278.

[17] E.A. Carver, R. Jiang, Y. Lan, K.F. Oram, T. Gridley, The mouse snail gene encodes a key regulator of the epithelial-mesenchymal transition, Mol. Cell. Biol. 21 (2001) 8184-8188.

[18] A. Eger, K. Aigner, S. Sonderegger, B. Dampier, S. Oehler, M. Schreiber, G. Berx, A. Cano, H. Beug, R. Foisner, DeltaEF1 is a transcriptional repressor of E-cadherin and regulates epithelial plasticity in breast cancer cells, Oncogene 24 (2005) 2375-2385.

[19] M.P. Stemmler, A. Hecht, R. Kemler, E-cadherin intron 2 contains cis-regulatory elements essential for gene expression, Development 132 (2005) 965-976.

[20] M.P. Stemmler, A. Hecht, B. Kinzel, R. Kemler, Analysis of regulatory elements of E-cadherin with reporter gene constructs in transgenic mouse embryos, Dev. Dyn. 227 (2003) 238-245.

[21] M. Werth, K. Walentin, A. Aue, J. Schonheit, A. Wuebken, N. Pode-Shakked, L. Vilianovitch, B. Erdmann, B. Dekel, M. Bader, J. Barasch, F. Rosenbauer, F.C. Luft, K.M. Schmidt-Ott, The transcription factor grainyhead-like 2 regulates the molecular composition of the epithelial apical junctional complex, Development 137 (2010) 3835-3845.

[22] H. Hagege, P. Klous, C. Braem, E. Splinter, J. Dekker, G. Cathala, W. de Laat, T. Forne, Quantitative analysis of chromosome conformation capture assays (3C-qPCR), Nat. Protoc. 2 (2007) 1722-1733.

[23] H. Alotaibi, E. Yaman, D. Salvatore, V. Di Dato, P. Telkoparan, R. Di Lauro, U.H. Tazebay, Intronic elements in the $\mathrm{Na}+/ \mathrm{I}$ - symporter gene (NIS) interact with retinoic acid receptors and mediate initiation of transcription, Nucleic Acids Res. 38 (2010) 3172-3185.

[24] D. Boffelli, J. McAuliffe, D. Ovcharenko, K.D. Lewis, I. Ovcharenko, L. Pachter, E.M. Rubin, Phylogenetic shadowing of primate sequences to find functional regions of the human genome, Science 299 (2003) 1391-1394.

[25] C. Mayor, M. Brudno, J.R. Schwartz, A. Poliakov, E.M. Rubin, K.A. Frazer, L.S. Pachter, I. Dubchak, VISTA: visualizing global DNA sequence alignments of arbitrary length, Bioinformatics 16 (2000) 1046-1047.

[26] K. Quandt, K. Frech, H. Karas, E. Wingender, T. Werner, MatInd and MatInspector: new fast and versatile tools for detection of consensus matches in nucleotide sequence data, Nucleic Acids Res. 23 (1995) 4878-4884.

[27] Y. Rifat, V. Parekh, T. Wilanowski, N.R. Hislop, A. Auden, S.B. Ting, J.M. Cunningham, S.M. Jane, Regional neural tube closure defined by the Grainy head-like transcription factors, Dev. Biol. 345 (2010) 237-245.

[28] M.S. Almeida, S.J. Bray, Regulation of post-embryonic neuroblasts by Drosophila Grainyhead, Mech. Dev. 122 (2005) 1282-1293.

[29] S.B. Ting, T. Wilanowski, A. Auden, M. Hall, A.K. Voss, T. Thomas, V. Parekh, J.M. Cunningham, S.M. Jane, Inositol- and folate-resistant neural tube defects in mice lacking the epithelial-specific factor Grhl-3, Nat. Med. 9 (2003) 1513-1519.

[30] T. Wilanowski, J. Caddy, S.B. Ting, N.R. Hislop, L. Cerruti, A. Auden, L.L. Zhao, S. Asquith, S. Ellis, R. Sinclair, J.M. Cunningham, S.M. Jane, Perturbed desmosomal cadherin expression in grainy head-like 1-null mice, EMBO J. 27 (2008) 886-897.

[31] M.A. Battle, G. Konopka, F. Parviz, A.L. Gaggl, C. Yang, F.M. Sladek, S.A. Duncan, Hepatocyte nuclear factor 4alpha orchestrates expression of cell adhesion proteins during the epithelial transformation of the developing liver, Proc. Natl. Acad. Sci. U. S. A. 103 (2006) 8419-8424.

[32] J.C. Wang, J.M. Stafford, D.K. Granner, SRC-1 and GRIP1 coactivate transcription with hepatocyte nuclear factor 4, J. Biol. Chem. 273 (1998) 30847-30850.

[33] M.J. Barrero, S. Malik, Two functional modes of a nuclear receptor-recruited arginine methyltransferase in transcriptional activation, Mol. Cell 24 (2006) 233-243.

[34] D. Yusuf, S.L. Butland, M.I. Swanson, E. Bolotin, A. Ticoll, W.A. Cheung, X.Y. Zhang, C.T. Dickman, D.L. Fulton, J.S. Lim, J.M. Schnabl, O.H. Ramos, M. Vasseur-Cognet, C.N. de Leeuw, E.M. Simpson, G.U. Ryffel, E.W. Lam, R. Kist, M.S. Wilson, R. MarcoFerreres, J.J. Brosens, L.L. Beccari, P. Bovolenta, B.A. Benayoun, L.J. Monteiro, H.D. Schwenen, L. Grontved, E. Wederell, S. Mandrup, R.A. Veitia, H. Chakravarthy, P.A. Hoodless, M.M. Mancarelli, B.E. Torbett, A.H. Banham, S.P. Reddy, R.L. Cullum, M. Liedtke, M.P. Tschan, M. Vaz, A. Rizzino, M. Zannini, S. Frietze, P.J. Farnham, A. Eijkelenboom, P.J. Brown, D. Laperriere, D. Leprince, T. de Cristofaro, K.L. Prince, M. Putker, L. del Peso, G. Camenisch, R.H. Wenger, M. Mikula, M. Rozendaal, S. Mader, J. Ostrowski, S.J. Rhodes, C. Van Rechem, G. Boulay, S.W. Olechnowicz, M.B. Breslin, M.S. Lan, K.K. Nanan, M. Wegner, J. Hou, R.D. Mullen, S.C. Colvin, P.J. Noy, C.F. Webb, M.E. Witek, S. Ferrell, J.M. Daniel, J. Park, S.A. Waldman, D.J. Peet, M. Taggart, P.S. Jayaraman, J.J. Karrich, B. Blom, F. Vesuna, H. O'Geen, Y. Sun, R.M. Gronostajski, M.W. Woodcroft, M.R. Hough, E. Chen, G.N. Europe-Finner, M. Karolczak-Bayatti, J. Bailey, O. Hankinson, V. Raman, D.P. LeBrun, S. Biswal, C.J. Harvey, J.P. DeBruyne, J.B. Hogenesch, R.F. Hevner, C. Heligon, X.M. Luo, M.C. Blank, K.J. Millen, D.S. Sharlin, D. Forrest, K. Dahlman-Wright, C. Zhao, Y. Mishima, S. Sinha, R. Chakrabarti, E. Portales-Casamar, F.M. Sladek, P.H. Bradley, W.W. Wasserman, The transcription factor encyclopedia, Genome Biol. 13 (2012) R24.

[35] S.F. Boj, M. Parrizas, M.A. Maestro, J. Ferrer, A transcription factor regulatory circuit in differentiated pancreatic cells, Proc. Natl. Acad. Sci. U. S. A. 98 (2001) $14481-14486$.

[36] H. Nakhei, A. Lingott, I. Lemm, G.U. Ryffel, An alternative splice variant of the tissue specific transcription factor HNF4alpha predominates in undifferentiated murine cell types, Nucleic Acids Res. 26 (1998) 497-504

[37] F. Ishikawa, K. Nose, M. Shibanuma, Downregulation of hepatocyte nuclear factor4alpha and its role in regulation of gene expression by TGF-beta in mammary epithelial cells, Exp. Cell Res. 314 (2008) 2131-2140.

[38] I. Bedzhov, E. Liszewska, B. Kanzler, M.P. Stemmler, Igf1r signaling is indispensable for preimplantation development and is activated via a novel function of E-cadherin, PLoS Genet. 8 (2012) e1002609. 
[39] L. Larue, M. Ohsugi, J. Hirchenhain, R. Kemler, E-cadherin null mutant embryos fail to form a trophectoderm epithelium, Proc. Natl. Acad. Sci. U. S. A. 91 (1994) 8263-8267.

[40] D. Riethmacher, V. Brinkmann, C. Birchmeier, A targeted mutation in the mouse E-cadherin gene results in defective preimplantation development, Proc. Natl. Acad. Sci. U. S. A. 92 (1995) 855-859.

[41] M.P. Stemmler, I. Bedzhov, A Cdh1HA knock-in allele rescues the Cdh1 - / - phenotype but shows essential Cdh1 function during placentation, Dev. Dyn. 239 (2010) 2330-2344.

[42] A. Auden, J. Caddy, T. Wilanowski, S.B. Ting, J.M. Cunningham, S.M. Jane, Spatial and temporal expression of the Grainyhead-like transcription factor family during murine development, Gene Expr. Patterns 6 (2006) 964-970.

[43] T. Wilanowski, A. Tuckfield, L. Cerruti, S. O'Connell, R. Saint, V. Parekh, J. Tao, J.M. Cunningham, S.M. Jane, A highly conserved novel family of mammalian developmental transcription factors related to Drosophila grainyhead, Mech. Dev. 114 (2002) 37-50.

[44] S.J. Bray, F.C. Kafatos, Developmental function of Elf-1: an essential transcription factor during embryogenesis in Drosophila, Genes Dev. 5 (1991) 1672-1683.

[45] C. Pyrgaki, A. Liu, L. Niswander, Grainyhead-like 2 regulates neural tube closure and adhesion molecule expression during neural fold fusion, Dev. Biol. 353 (2011) $38-49$.

[46] S.A. Duncan, K. Manova, W.S. Chen, P. Hoodless, D.C. Weinstein, R.F. Bachvarova, J.E. Darnell Jr., Expression of transcription factor HNF-4 in the extraembryonic endoderm, gut, and nephrogenic tissue of the developing mouse embryo: HNF-4 is a marker for primary endoderm in the implanting blastocyst, Proc. Natl. Acad. Sci. U. S. A. 91 (1994) 7598-7602.

[47] W.S. Chen, K. Manova, D.C. Weinstein, S.A. Duncan, A.S. Plump, V.R. Prezioso, R.F. Bachvarova, J.E. Darnell Jr., Disruption of the HNF-4 gene, expressed in visceral endoderm, leads to cell death in embryonic ectoderm and impaired gastrulation of mouse embryos, Genes Dev. 8 (1994) 2466-2477.

[48] W.D. Garrison, M.A. Battle, C. Yang, K.H. Kaestner, F.M. Sladek, S.A. Duncan, Hepatocyte nuclear factor 4alpha is essential for embryonic development of the mouse colon, Gastroenterology 130 (2006) 1207-1220.

[49] J. Shi, W.A. Whyte, C.J. Zepeda-Mendoza, J.P. Milazzo, C. Shen, J.S. Roe, J.L. Minder, F. Mercan, E. Wang, M.A. Eckersley-Maslin, A.E. Campbell, S. Kawaoka, S. Shareef, Z Zhu, J. Kendall, M. Muhar, C. Haslinger, M. Yu, R.G. Roeder, M.H. Wigler, G.A. Blobel, J. Zuber, D.L. Spector, R.A. Young, C.R. Vakoc, Role of SWI/SNF in acute leukemia maintenance and enhancer-mediated Myc regulation, Genes Dev. 27 (2013) 2648-2662.

[50] A. Sanyal, B.R. Lajoie, G. Jain, J. Dekker, The long-range interaction landscape of gene promoters, Nature 489 (2012) 109-113.

[51] E. Bertolino, H. Singh, POU/TBP cooperativity: a mechanism for enhancer action from a distance, Mol. Cell 10 (2002) 397-407.

[52] C.R. Vakoc, D.L. Letting, N. Gheldof, T. Sawado, M.A. Bender, M. Groudine, M.J. Weiss J. Dekker, G.A. Blobel, Proximity among distant regulatory elements at the betaglobin locus requires GATA-1 and FOG-1, Mol. Cell 17 (2005) 453-462.

[53] K. Yun, J.S. So, A. Jash, S.H. Im, Lymphoid enhancer binding factor 1 regulates transcription through gene looping, J. Immunol. 183 (2009) 5129-5137. 